\title{
Priapisme veineux : traitement et pronostic fonctionnel. A propos de 26 cas
}

\author{
Mourad HADJ SLIMEN, Hammadi FAKHFAKH, Mohamed GASSARA, \\ Sofiane KECHAOU, Ali BAHLOUL, Mohamed Nabil MHIRI
}

Service d'Urologie, CHU Habib Bourguiba, Sfax, Tunisie

\section{RESUME}

Introduction : le priapisme est une érection anormalement prolongée, douloureuse et irréductible survenant en dehors de toute stimulation sexuelle. II continue de poser des problèmes, tant sur le plan thérapeutique, que pronostique. Les auteurs se proposent d'étudier les différents aspects étiopathogéniques, thérapeutiques, et évolutifs de cette affection.

Matériel et méthodes : 26 cas de priapisme veineux d'étiologies variées ont été colligés en 23 ans (1985-2007). II s'agit de 22 adultes et de 4 enfants (âge moyen : 32 ans). La prise en charge s'est faite sans délai en fonction de l'étiologie et du délai de consultation (variant de 16 heures à 15 jours).

Résultats : l'obtention d'une détumescence complète et irréversible au cours des premières 24 heures a été notée dans $84 \%$ des cas, parfois au prix de plusieurs procédés thérapeutiques successifs. L'évolution à distance a été appréciée chez 21 malades avec un recul moyen de 2 ans. Aucun cas de récidive tardive n'a été observé. La récupération d'une fonction érectile normale a été observée dans $42,9 \%$ des cas.

Conclusion : le priapisme veineux doit être considéré comme une urgence uro-andrologique majeure. Le traitement médical peut être efficace dans les formes précoces mais il ne faut pas laisser passer l'heure de la chirurgie. Le traitement de tout facteur de risque doit être mené en parallèle afin de prévenir les récidives.

Mots clés : priapisme, érection pharmacologique, étiologie, traitement, évolution

\section{INTRODUCTION}

Le priapisme, véritable urgence andrologique, continue de poser des problèmes tant sur le plan thérapeutique que pronostique. Le diagnostic de cette affection est évident, cependant, il souffre souvent du côté tabou que représente la sexualité sous nos climats, responsable souvent d'un retard important de consultation.

Il existe 2 types de priapisme : le priapisme à haut débit (ou artériel), rare, indolore, n'évoluant jamais vers l'ischémie, qui est provoqué par une fistule artério-caverneuse acquise responsable d'un excès du flux artériel entrant ; et le priapisme de stase (ou veineux), le plus fréquent et le plus urgent, qui est la conséquence directe d'une anomalie du drainage caverneux.

Les étiologies sont multiples et variées avec une place prépondérante des formes médicamenteuses iatrogènes.

De nouvelles approches thérapeutiques, en particulier pharmacologiques ont vu le jour, mais la chirurgie occupe une place prépondérante et ceci en raison du retard fréquent de prise en charge. Le pronostic reste toutefois sombre avec plus de $50 \%$ de dysfonction érectile majeure chez des patients souvent jeunes avec de lourdes conséquences sociales et psychologiques.

Ces différents problèmes sont étudiés à la lumière d'une série personnelle de 26 cas de priapisme veineux avec revue de la littérature.

\section{Correspondance :}

Dr Mourad HADJ SLIMEN - Service d'Urologie - CHU Habib Bourguiba, 3029, Sfax, Tunisie - Tel 0021698660667 Fax 0021674243427 - Email mourad.hadjslimen@laposte.net 


\section{PATIENTS ET METHODES}

\section{Patients}

Entre janvier 1985 et décembre 2007, vingt six patients ont été pris en charge pour priapisme veineux et étudiés de façon rétrospective. L'âge moyen des patients était de 32 ans avec des extrêmes de 18 mois et 60 ans. II s'agissait de 22 adultes, et de 4 enfants âgés de 18 mois à 16 ans.

Le délai moyen de consultation a été très variable allant de 16 heures à 15 jours (médiane : 5 jours). Plus des deux tiers des patients ont consulté au-delà de $\mathbf{4 8}$ heures. Seuls trois patients se sont présentés avant la $36^{\text {ème heure après }}$ l'apparition de l'érection anormale. La douleur pénienne a été présente chez la quasi-totalité des patients et a motivé la consultation en plus du caractère inefficace des rapports sexuels.

Une étiologie ou un facteur favorisant ont été retrouvés chez 14 des 22 adultes $(63,6 \%)$, dominés par les causes médicamenteuses, notamment les psychotropes neuroleptiques (9 cas) (Tableau 1). Une notion de traumatisme pénoscrotal de moyenne gravité (chute à califourchon), survenant quelques jours avant l'installation du priapisme, a été rapportée par un patient sans véritable preuve de relation de cause à effet puisque l'examen a orienté plutôt vers un priapisme de stase (douleur pénienne, corps caverneux rigides et gland flasque). Pour les 8 patients restants $(30,7 \%)$, aucun facteur pathologique, pouvant interférer avec l'érection, n'a été retrouvé, avec toutefois notion d'érections prolongées spontanément résolutives dans 2 cas.

Un facteur étiologique a été retrouvé chez tous les enfants, représenté par une hémopathie (2 cas), une envenimation scorpionique grave (1 cas), et une administration intramusculaire de hCG (Gonadotrophine Chorionique humaine) dans le cadre d'un traitement hormonal pour cryptorchidie chez un nourrisson de 18 mois (2ème injection hebdomadaire de 500 Ul de hCG).

\section{Schéma thérapeutique}

Tous les patients ont été pris en charge en urgence sauf le patient porteur d'une leucémie, décédé suite à une hémorragie cérébro-méningée avant tout traitement spécifique. Plusieurs procédés thérapeutiques ont été utilisés, selon un schéma d'escalade, en fonction de l'étiologie et du délai de consultation (Tableau 2) :

- Le traitement médical : une injection intra caverneuse (IIC) de drogue alpha stimulante (1mg d'éphédrine dilué dans $10 \mathrm{ml}$ de sérum physiologique) a été réalisée chez 3 patients présentant un priapisme iatrogène. Celle-ci a été réalisée au bloc opératoire sous monitorage et surveillance hémodynamique.

- La ponction caverneuse : une ponction évacuatrice des corps caverneux a été effectuée d'emblée chez 7 patients, et après échec du traitement médical dans 3 cas. La ponction a été réalisée, sous anesthésie générale, à l'aide d'un cathéter périphérique 14G type cathlon, avec évacuation de sang noirâtre jusqu'à obtention de la détumescence.
- Le traitement chirurgical : 20 patients ont été traités chirurgicalement, d'emblée dans 15 cas devant un délai de consultation dépassant, dans tous les cas, 3 jours, et après échec des méthodes médicales dans les 5 cas restants. Plusieurs techniques opératoires ont été utilisées, à savoir :

a) Une fistule spongio-caverneuse trans-glandulaire à ciel fermé selon la technique de Winter dans 2 cas (à l'aide d'un trocart $14 \mathrm{G}$ réalisant un tunnel entre le gland et chacun des corps caverneux).

b) Une anastomose spongio-caverneuse unilatérale à ciel ouvert par voie trans-glandulaire selon la technique d'Al Ghorab dans 7 cas.

c) Une anastomose caverno-spongieuse proximale au niveau de l'angle péno-scrotal selon la technique de Cukier dans 4 cas.

d) Enfin, une anastomose caverno-spongieuse proximale par voie périnéale selon la technique de Quackels dans 7 cas.

Quelque soit la technique utilisée, une large anastomose entre le corps caverneux et le corps spongieux a été réalisée après évacuation du sang noirâtre emprisonné dans les corps caverneux et obtention d'une détumescence complète immédiate.

Tous les patients ont été mis systématiquement sous traitement médical anti-érectogène. Le séjour postopératoire moyen a été de 3 jours.

\section{RESULTATS}

\section{Evolution immédiate}

L'obtention d'une détumescence complète et irréversible au cours des premières 24 heures a été notée chez 20 patients parmi les malades traités ( $84 \%)$. Toutefois, elle a nécessité, dans 4 cas, le recours à plusieurs procédés thérapeutiques successifs (Figure 1 et Tableau 2).

Nous avons relevé un cas de récidive précoce survenant 3 jours après une ponction intra-caverneuse réalisée en première intention pour un priapisme idiopathique évoluant depuis 2 jours avec résultat initial satisfaisant. Ce patient a bénéficié, avec succès, d'une anastomose caverno-spongieuse unilatérale par voie périnéale.

Pour les 4 patients restants, une détumescence immédiate mais incomplète a été obtenue, mais l'évolution a été marquée par une récidive rapide d'un état de tumescence de plus en plus important se prolongeant au-delà de 24 heures. Tous ces patients ont été repris chirurgicalement, avec réalisation d'une anastomose caverno-spongieuse bilatérale (2 transglandulaires et 2 périnéales). L'évolution ultérieure a été favorable chez 3 patients. Enfin, tous les moyens thérapeutiques ont été jugés inefficaces chez le dernier patient (priapisme drépanocytaire évoluant depuis 5 jours).

Quelques complications postopératoires immédiates ont été observées ; à savoir un hématome sous-cutané (4 cas), une surinfection locale de la plaie (1 cas), et une fistule urinaire périnéale faisant suite à une anastomose caverno-spongieuse 
Tableau 1 : les différentes étiologies de priapisme retrouvées dans notre série.

\begin{tabular}{|c|c|c|c|}
\hline Etiologies & Age & $\begin{array}{l}\text { Nombre } \\
\text { de cas }\end{array}$ & $\%$ \\
\hline $\begin{array}{c}\text { Neuroleptiques: } \\
\text { - Halopéridol } \\
\text { - Fluphénazine } \\
\text { - Périciazine } \\
\text { - Thiopropérazine }\end{array}$ & adulte & $\begin{array}{l}9 \\
5 \\
2 \\
1 \\
1\end{array}$ & 34,6 \\
\hline Injection de hCG & nourrisson & 1 & 3,9 \\
\hline Envenimation scorpionique grave & enfant & 1 & 3,9 \\
\hline $\begin{array}{l}\text { Hématologique: } \\
\text { - Drépanocytose } \\
\text { - Leucémie }\end{array}$ & $\begin{array}{c}1 \text { enfant }+1 \text { adulte } \\
\text { enfant }\end{array}$ & $\begin{array}{l}3 \\
2 \\
1\end{array}$ & 11,5 \\
\hline Papavérine (injection intracaverneuse) & adulte & 3 & 11,5 \\
\hline Traumatisme péno-scrotal & adulte & 1 & 3,9 \\
\hline Idiopathique & adulte & 8 & 30,7 \\
\hline Total & & 26 & 100 \\
\hline
\end{tabular}

hCG : Gonadotrophine Chorionique humaine.

Tableau 2 : résultats immédiats en fonction des différentes méthodes thérapeutiques.

\begin{tabular}{|c|c|c|c|c|c|}
\hline \multirow{2}{*}{ Traitement } & \multirow{2}{*}{$\begin{array}{l}\text { Nbre de } \\
\text { cas }\end{array}$} & \multirow{2}{*}{$\begin{array}{l}\text { Délai thérapeutique } \\
\text { moyen (jours) }\end{array}$} & \multicolumn{3}{|c|}{ Résultats immédiats } \\
\hline & & & Succès & Echec & Récidive \\
\hline IIC d'éphédrine & 3 & 2 & 0 & 3 & - \\
\hline $\begin{array}{l}\text { Ponction intracaverneuse } \pm \\
\text { injection intracaverneuse }\end{array}$ & 10 & $\begin{array}{c}1,5 \\
2,5 \\
2 \\
\end{array}$ & $\begin{array}{l}5 \\
- \\
- \\
\end{array}$ & $\begin{array}{r}- \\
4 \\
- \\
\end{array}$ & $\begin{array}{l}- \\
- \\
1\end{array}$ \\
\hline $\begin{array}{l}\text { Fistule spongio-caverneuse } \\
\text { à ciel fermé (Winter) }\end{array}$ & 2 & $\begin{array}{l}5 \\
3 \\
\end{array}$ & $\begin{array}{l}1 \\
- \\
\end{array}$ & $\overline{1}$ & 0 \\
\hline $\begin{array}{l}\text { Shunt spongio-caverneux } \\
\text { trans-glandulaire (Al Ghorab) }\end{array}$ & 7 & $\begin{array}{l}6 \\
3\end{array}$ & 5 & $\overline{2}$ & 0 \\
\hline $\begin{array}{l}\text { Anastomose caverno-spongieuse } \\
\text { périnéale }\end{array}$ & 11 & $\begin{array}{l}5 \\
4\end{array}$ & 10 & $\overline{1}$ & 0 \\
\hline
\end{tabular}

IIC : injection intra-caverneuse.

Tableau 3 : résultats thérapeutiques (fonction érectile) en fonction de l'âge et du délai de prise en charge.

\begin{tabular}{|c|c|c|c|c|c|}
\hline \multirow{2}{*}{ Résultat } & \multicolumn{2}{|c|}{ Age (ans) } & \multirow{2}{*}{$\begin{array}{c}\text { Délai thérapeutique } \\
\text { (jours) }\end{array}$} & \multirow{2}{*}{$\begin{array}{l}\text { Nbre de } \\
\text { cas }\end{array}$} & \multirow{2}{*}{$\begin{array}{l}\text { Fréquence } \\
\qquad(\%)\end{array}$} \\
\hline & Moyenne & Extrêmes & & & \\
\hline Succès & 33,3 & $13-49$ & $1,5-4(M: 2,25)$ & 9 & 42,9 \\
\hline Partiel & 34,7 & $20-47$ & $3-6(M: 5)$ & 4 & 19 \\
\hline Echec & 44,1 & $27-60$ & $3-10(M: 6,6)$ & 8 & 38,1 \\
\hline
\end{tabular}




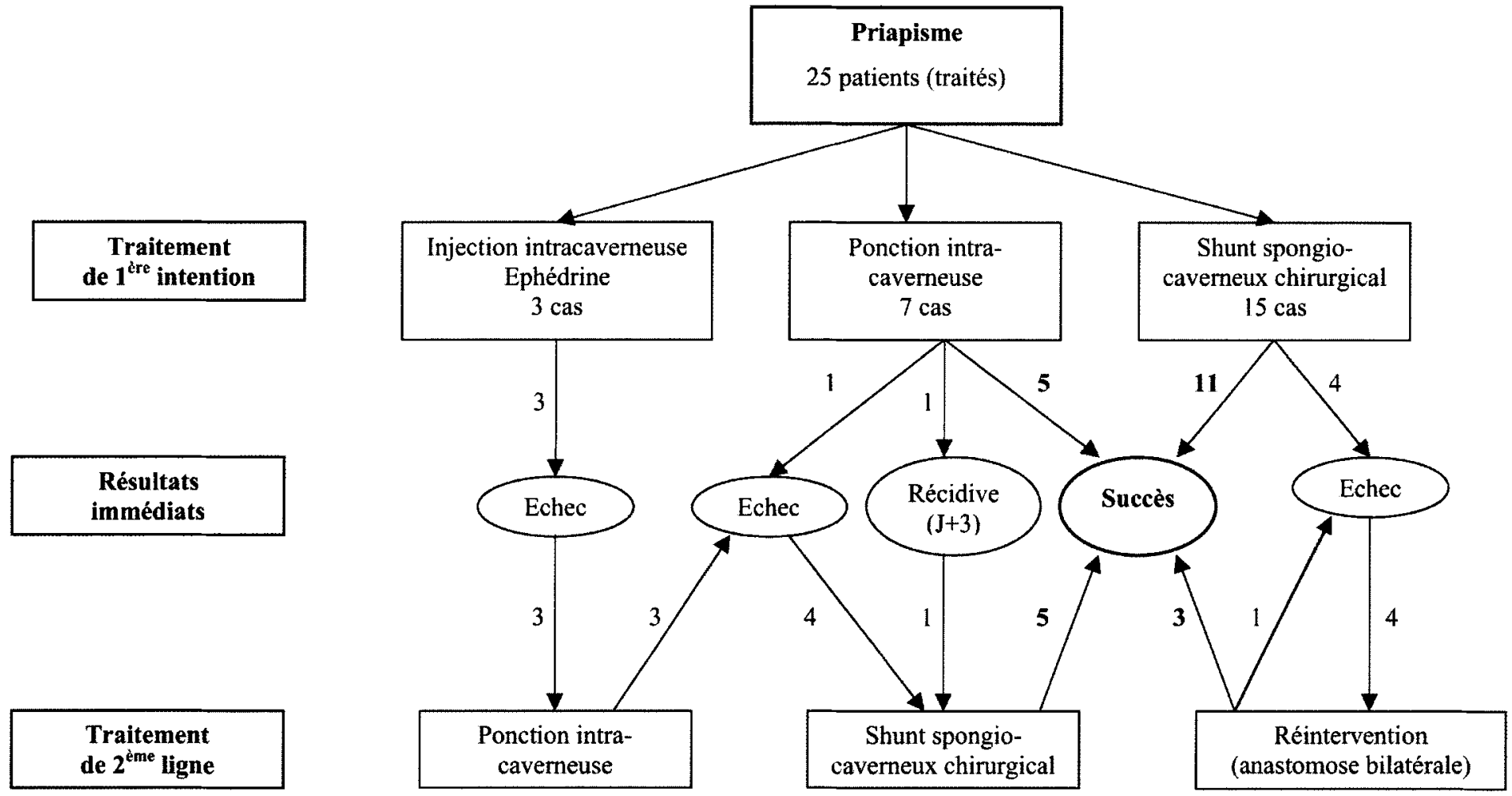

Figure 1 : schéma thérapeutique adopté dans notre série et résultats immédiats.

périnéale ( 1 cas). Toutes ces complications ont évolué favorablement sous traitement médical (anti-inflammatoire, antibiotique, et drainage urinaire prolongé).

\section{Evolution à long terme}

L'évolution à distance a pu être appréciée chez 21 patients avec un recul moyen de 2 ans ( 3 mois à 10 ans). Les 4 malades restants ont été perdus de vue. Aucun cas de récidive tardive de priapisme n'a été observé.

En ce qui concerne la fonction érectile, neuf malades $(42,9 \%)$ ont retrouvé une activité sexuelle satisfaisante avec des érections rigides et sans courbure. L'âge moyen de ces patients était de 33 ans. Le délai moyen de consultation était de 54 heures avec des extrêmes de 36 et 72 heures. Le traitement a été dans tous les cas chirurgical, dont 5 anastomoses caverno-spongieuses par voie périnéale, avec succès immédiat et durable et sans aucune complication précoce.

Quatre patients (19\%) ont rapporté une dysfonction érectile marquée décrite comme de simples états de tumescence faible avec un potentiel érectile conservé au niveau de la base de la verge, peu intense, permettant des rapports sexuels rares, éloignés, et peu satisfaisants pour le couple. Ces patients ont gardé des éjaculations, un orgasme, et de rares érections nocturnes et matinales spontanées. L'examen de ces patients a révélé la présence d'une fibrose peu étendue des corps caverneux avec une rétraction minime de la verge et une perte de son élasticité. L'âge de ces patients était comparable à celui du premier groupe (moyenne $=34$ ans), mais le délai thérapeutique était plus tardif (moyenne $=5$ jours). Le traitement était également chirurgical dans tous les cas, mais réalisé en 2 temps chez deux malades devant l'échec immédiat du procédé utilisé en première intention.

Enfin, 8 malades $(38,1 \%)$ ont signalé une dysfonction érectile majeure avec absence totale d'érection et impossibilité de tout rapport sexuel et d'éjaculation malgré une libido conservée. L'examen a montré une fibrose étendue des corps caverneux entraînant une rétraction importante de la verge et une diminution importante de l'extensibilité pénienne. L'âge moyen de ces patients était plus avancé (44 ans) avec des extrêmes de 27 et 60 ans. Le délai de consultation était plus important allant de 3 à 10 jours (moyenne de 6,6 jours). Les méthodes thérapeutiques utilisées pour ce groupe ont été multiples avec recours à une chirurgie en deux temps à 2 reprises.

Les résultats thérapeutiques ont été étudiés en fonction de plusieurs paramètres, dont l'âge des malades et le délai de prise en charge (Tableau 3), l'étiologie du priapisme (Tableau 4) et les différentes modalités thérapeutiques (Tableau 5).

\section{DISCUSSION}

Le nombre restreint des cas de priapisme publiés dans la littérature et celui de notre série témoignent de la rareté de cette affection. Son incidence est d'environ 1,5 pour 100.000 personnes par an $[5,8]$. Le priapisme survient à tout âge 
Tableau 4 : résultats thérapeutiques (fonction érectile) en fonction de l'étiologie du priapisme.

\begin{tabular}{|c|c|c|c|c|c|}
\hline \multirow{2}{*}{ Etiologies } & \multirow{2}{*}{ Nombre de cas } & \multicolumn{3}{|c|}{ Résultats } & \multirow{2}{*}{$\begin{array}{l}\text { Perdus de } \\
\text { vue }\end{array}$} \\
\hline & & Succès & Partiel & Echec & \\
\hline Neuroleptiques & 9 & 5 & 1 & 2 & 1 \\
\hline Hémopathie & 2 & - & 2 & - & - \\
\hline Post traumatique & 1 & - & - & 1 & - \\
\hline Papavérine & 3 & - & - & 3 & - \\
\hline hCG & 1 & - & - & - & 1 \\
\hline Envenimation scorpionique & 1 & - & - & - & 1 \\
\hline Idiopathique & 8 & 4 & 1 & 2 & 1 \\
\hline Total & 25 & 9 & 4 & 8 & 4 \\
\hline
\end{tabular}

Tableau 5 : résultats thérapeutiques (fonction érectile) en fonction du type d'intervention.

\begin{tabular}{|c|c|c|c|c|c|c|}
\hline \multirow{2}{*}{ Type d'intervention } & \multirow{2}{*}{$\begin{array}{c}\text { Nbre } \\
\text { cas }\end{array}$} & \multirow{2}{*}{$\begin{array}{c}\text { Délai } \\
\text { (jours) }\end{array}$} & \multicolumn{3}{|c|}{ Résultats } & \multirow{2}{*}{$\begin{array}{c}\text { Perdus de } \\
\text { vue }\end{array}$} \\
\hline & & & Succès & Partiel & Echec & \\
\hline Ponction caverneuse & 5 & 1,3 & 2 & 0 & 2 & 1 \\
\hline Technique de Winter & 1 & 5 & - & 1 & - & - \\
\hline Technique d'Al Ghorab & 8 & 5,5 & 2 & 2 & 3 & 1 \\
\hline Anastomose périnéale & 11 & 5 & 5 & 1 & 3 & 2 \\
\hline Total & 25 & & 9 & 4 & 8 & 4 \\
\hline
\end{tabular}

depuis les nouveaux-nés jusqu'aux vieillards. L'incidence augmente à 2,9 pour 100.000 personnes par an pour les hommes de plus de 40 ans [11]. Cette incidence tend à augmenter encore avec l'accroissement de prescription des médicaments de la dysfonction érectile, particulièrement des injections intra caverneuses [3]. Classiquement, il existe deux pics de fréquence : entre 5 et 10 ans chez les enfants, surtout en zones d'endémie drépanocytaire dans certains pays d'Afrique $[9,10,15]$, et entre 20 et 50 ans chez l'adulte, chez qui, les causes pharmacologiques sont prédominantes. Dans notre série, $84,6 \%$ des malades étaient des adultes âgés entre 20 et 60 ans (moyenne : 34,4 ans), pour le reste, il s'agissait de 3 enfants à un âge pré-pubaire $(2$ cas d'hémopathie et une envenimation scorpionique) et d'un bébé de 18 mois recevant un traitement hormonal pour une cryptorchidie.

\section{Physiopathologie du priapisme veineux $[1,3,18]$}

Le priapisme veineux (de stase ou de bas débit) reflète une anomalie de la détumescence, ou plus précisément, du drainage caverneux, qui peut être bloqué plus ou moins complètement, par deux mécanismes. Le premier, neuromusculaire, est induit par des substances ayant un effet alpha-bloquant (médicaments, toxiques, venin de scorpion...) ou consécutif à l'injection intra caverneuse de produits érectogènes dans le traitement de la dysfonction érectile, responsables d'un blocage de la recontraction du muscle lisse érectile caverneux.

Le second mécanisme, hématologique, est du à une hyperviscosité du sang intracaverneux secondaire à certaines maladies hématologiques (drépanocytose, leucémies, polyglobulies, thrombocytémies...) ou parfois d'origine iatrogène (solutés hyperlipidiques). La recontraction caverneuse est possible, mais peu ou pas efficace, en raison du ralentissement de la vidange caverneuse à travers les veines émissaires.

Dans les deux cas, le priapisme veineux doit être considéré 
comme une urgence urologique en raison de son risque spécifique d'ischémie aiguë des corps caverneux significativement corrélée à la durée du priapisme. Si cet état se prolonge au-delà de plusieurs heures, la stase veineuse entraîne une souffrance cellulaire de type anoxique (acidose, hypoxie et hypercapnie à la gazométrie caverneuse) avec des lésions tissulaires progressives des fibres musculaires lisses érectiles des corps caverneux. Après 24 heures, une nécrose tissulaire responsable d'une fibrose séquellaire peut survenir, le risque de séquelles est majeur après 36 heures.

\section{Etiologies du priapisme veineux}

L'étiopathogénie est multifactorielle (Tableau 6), on oppose classiquement les priapismes secondaires aux formes idiopathiques qui représentent entre 30 et $70 \%$ des cas selon les séries $[1,3,5,9,14]$. Les causes médicamenteuses sont les plus fréquentes, retrouvées dans environ $30 \%$ des cas [4]. Les hémopathies représentent la cause majeure de priapisme chez l'enfant. La drépanocytose est responsable de 2 à $5 \%$ des priapismes chez l'adulte et de 38 à $42 \%$ chez l'enfant [18]. Le priapisme iatrogène secondaire aux IIC de drogues érectogènes a considérablement diminué depuis l'avènement de nouvelles drogues comme la Prostaglandine E1 [6]. Dans notre série, un facteur étiologique a été retrouvé dans $69,3 \%$ des cas. Les formes idiopathiques $(30,7 \%)$ ont été observées uniquement chez les adultes.

\section{Diagnostic}

- La survenue de priapisme se fait de façon inopinée, brutale et souvent très rapide sans stimulation sexuelle. Le caractère douloureux et persistant de l'érection amène le plus souvent à consulter. Les délais de consultation sont très variables allant de quelques heures à quelques semaines. Ce délai de consultation est important puisqu'il conditionne le choix thérapeutique et le pronostic. Dans notre série, un retard considérable de consultation a été observé (entre 3 et 15 jours dans $70 \%$ des cas).

- Le diagnostic de priapisme veineux repose avant tout sur la clinique [3]. Il est, en effet, évident dès l'inspection avec une verge tendue, en érection irréductible, plaquée contre l'abdomen. Seuls les corps caverneux sont indurés « en fer " jusqu'à leur portion périnéale ; le corps spongieux ainsi que le gland ne sont pas concernés par cette induration et restent flasques. La douleur pénienne apparaît à partir de la 4 ème heure et devient de plus en plus intense, pouvant entraîner une insomnie et un sentiment d'angoisse $[1,20,21]$. Toute survenue d'une douleur, spontanée ou provoquée par la pression de la verge, a une valeur d'alarme. Elle reflète une souffrance tissulaire due à l'hyperpression intracaverneuse et/ou à la souffrance anoxique.

- La gazométrie du sang caverneux est le meilleur moyen pour évaluer la gravité de l'ischémie caverneuse $[1,3]$. Une $\mathrm{pO}_{2}<50 \mathrm{mmHg}$, une $\mathrm{pCO}_{2}>60 \mathrm{mmHg}$ et un $\mathrm{pH}<7,3$ témoignent déjà d'une souffrance anoxique. Une $\mathrm{pO}_{2}$ $<30 \mathrm{mmHg}$ reflète une souffrance sévère.

- Parallèlement, le bilan étiologique (hémogramme, myélogramme au moindre doute, imagerie spécifique) est indispensable pour faire cesser la stimulation pathologique et éviter la récidive immédiate ou à distance [18].

\section{Evolution}

Non traité, le priapisme peut évoluer initialement vers une accentuation progressive de la douleur qui diminue ensuite avec survenue de détumescence et installation d'une fibrose caverneuse irréversible responsable d'impuissance séquellaire [16]. Ceci impose une prise en charge précoce efficace et sans délais.

\section{Traitement du priapisme veineux}

Le traitement vise à redonner, le plus rapidement possible, un état de flaccidité durable des corps caverneux avant que ne s'installent des lésions tissulaires, afin de préserver ultérieurement la fonction érectile. Ce traitement comporte deux volets : un rétablissement physiologique ou chirurgical d'un drainage efficace des corps caverneux en plus d'un traitement étiologique qui doit être réalisé parallèlement, chaque fois que possible, pour faire cesser le facteur stimulant pathologique et éviter les récidives.

\section{Un schéma thérapeutique}

tenant compte essentiellement du délai de prise en charge, des signes de souffrance anoxique (représentés cliniquement par la douleur), et de l'étiologie, peut être proposé $[1,3,18$, 20]:

- En cas de priapisme indolore vu à un stade précoce (avant la 12ème heure) et secondaire aux injections intracaverneuses (IIC) de substances vaso-actives, certains "petits moyens" préalablement enseignés aux patients peuvent être essayés. II s'agit soit de moyens physiologiques par obtention d'une nouvelle éjaculation (rapport sexuel ou masturbation) ou un effort physique important (monter des étages en courant), ou de moyens artificiels tels que la réfrigération cutanée pénienne. Ces petits moyens peuvent suffire dans bon nombre de cas surtout s'ils sont utilisés précocement. En cas d'échec, la prise orale d'un alpha-stimulant (étiléphrine ou terbutaline) peut être essayée.

- En cas de priapisme indolore entre la 12 ème et la 24 ème heure, ou en cas d'échec des premiers moyens, la tendance actuelle est d'associer une ponction intracaverneuse et l'injection intracavemeuse d'une drogue alpha-stimulante (éphédrine ou phényléphrine). $\mathrm{Ce}$ traitement peut être réalisé sous anesthésie locale avec monitorage cardiovasculaire, et vise à décomprimer les corps caverneux, évacuer le sang de stase, et favoriser une recontraction directe des fibres musculaires des corps caverneux.

- En cas d'échec, de récidive, ou d'emblée en cas de suspicion d'anoxie (douleur et/ou délai $>24$ heures), la ponction des corps caverneux doit être faite en urgence sans délai associée ou non à une IIC de drogue alphastimulante. La ponction simple, dans ce cas, même si elle s'avère insuffisante, permet de soulager le patient jusqu'au temps chirurgical. La gazométrie du sang caverneux doit être réalisée en même temps que la ponction. Elle permet 


\begin{tabular}{lc}
\hline \hline IATROGENES & CAUSES HEMATOLOGIQUES \\
CAUSES MEDICAMENTEUSES & DREPANOCYTOSES \\
ANTICOAGULANTS (HEPARINE) & LEUCEMIES \\
ANTIHYPERTENSEURS & LEUCEMIE MYELOÏDE CHRONIQUE \\
ALPHABLOQUANTS (PRAZOSINE) & LEUCOSES AIGUES \\
DERIVES DE L'HYDRALAZINE & MYELOME MULTIPLE \\
VASODILATATEURS ARTERIELS & POLYGLOBULIES PRIMITIVES OU \\
INHIBITEURS CALCIQUES & SECONDAIRES \\
NEUROLEPTIQUES & THROMBOCYTEMIE ESSENTIELLE \\
PHENOTIAZINE & SYNDROME MYELOPROLIFERATIF \\
CHLORPOMAZINE & SPHEROCYTOSE CONGENITALE \\
HALOPERIDOL & THROMBOPHLEBITES INFECTIEUSES \\
FLUPHENAZINE & CAUSES NEOPLASIQUES \\
THIORIDAZINE & METASTASES CAVERNEUSES \\
ANTIDEPRESSEURS & (TUMEURS GENITOURINAIRES, \\
TRADOZONE & TUBE DIGESTIF) \\
DIAZEPAM & CANCER DE VERGE \\
PHENELZINE & CAUSES NEUROLOGIQUES \\
DROGUES ERECTOGENES & ACCIDENT VASCULAIRE CEREBRAL \\
PAPAVERINE (IIC) +++ & TRAUMATISME MEDULLAIRE \\
PHENTOLAMINE & TUMEURS CEREBRALES \\
PROSTAGLANDINE E1 (IIC OU & TUMEURS MEDULLAIRES \\
INTRA-URETRALE) & SCLEROSE EN PLAQUE \\
SILDÉNAFIL & NEUROTOXINES \\
HORMONES & VENIN DE SCORPION (SCORPAMINE) \\
GONADOTROPIN RELEASING- & VENIN D'ARAIGNEES \\
HORMONE & CAUSES METABOLIQUES \\
GONADOTROPHINE CHORIONIQUE & AMYLOSE \\
(hCG) & GOUTTE \\
TAMOXIFENE & DIABETE \\
TESTOSTERONE & INSUFFISANCE RENALE \\
PSYCHODYSLEPTIQUES & \\
ALCOOL & \\
COCAINE & \\
MARIJUANA & \\
CERTAINS ANESTHESIQUES & \\
NUTRITION PARENTERALE & \\
HYPERLIPIDIQUE & \\
\hline \hline & \\
\hline & \\
\hline & \\
\hline
\end{tabular}

IIC : injection intra-caverneuse. 
d'apprécier l'intensité de la souffrance et guidera le traitement ultérieur. En cas d'échec, assez fréquent après 24 heures ( $50 \%$ dans notre série), et en l'absence de critères d'anoxie sévère $(\mathrm{pO} 2>30 \mathrm{mmHg})$, la réinjection prudente d'alpha-stimulant est possible toutes les 10 à 15 minutes sous réserve de ne pas dépasser un délai d'une heure. En cas de nouvel échec ou de contre-indication à I'IIC, il est inutile d'attendre davantage, le traitement devient chirurgical.

Par contre, si la gazométrie montre d'emblée des signes d'anoxie sévère $\left(\mathrm{pH}<7,25, \mathrm{pO}_{2}<30 \mathrm{mmHg}\right.$ et $\mathrm{pCO}_{2}>$ $60 \mathrm{mmHg}$ ), le traitement chirurgical s'impose sans attente, avec création d'une fistule caverno-spongieuse qui facilite l'oxygénation caverneuse en évacuant le sang de stase.

- Le plus souvent, le traitement médical n'a pas fait preuve d'une grande efficacité, hormis certains cas de priapismes pharmacologiques vus dans les premières 48 heures, où les bons résultats avoisinent les $80 \%$ dans quelques séries $[1,3]$. Dans notre série, nous avons relevé un taux élevé d'échec des méthodes médicales imputé surtout au délai de prise en charge dépassant souvent les 48 heures.

- La chirurgie concerne les échecs de prise en charge médicale. Les techniques chirurgicales sont multiples, mais le principe est toujours le même. II s'agit de permettre au sang des aréoles caverneuses d'emprunter les voies de drainage veineux du corps spongieux ou du système veineux périphérique.

Quelque soit la technique chirurgicale utilisée, la détumescence est généralement obtenue, parfois au prix de plusieurs tentatives thérapeutiques successives avec un taux très faible de récidive précoce (un seul cas de récidive dans notre série survenant 3 jours après une ponction caverneuse réussie au départ et aucun cas de récidive après cure chirurgicale).

L'échec postopératoire immédiat, noté chez $20 \%$ de nos patients, impose une réintervention rapide avec réalisation d'une anastomose caverno-spongieuse bilatérale au mieux à la base de la verge. Le shunt distal ne semble pas utile en cas d'échec de l'anastomose proximale (1 double échec dans notre série). Il faudrait s'assurer de la durabilité de la détumescence obtenue au cours de la première intervention avec éventuellement d'emblée une bilatéralisation du shunt caverno-spongieux afin d'éviter une reprise chirurgicale.

Face aux techniques opératoires classiques et à leurs inconvénients, la ponction drainage balanique à ciel ouvert constitue une alternative nouvelle intéressante en raison de sa simplicité d'exécution et de la qualité des résultats obtenus. Elle permet une détumescence immédiate dans $80 \%$ des cas et, à distance, le taux global de succès (guérison et amélioration) est de $65,3 \%[10,11]$.

La prise en charge d'un éventuel facteur étiologique est primordiale et doit être menée de façon parallèle. Elle passe avant tout par l'arrêt, et plus ou moins, la substitution de toute drogue ou toxique en cause par un autre médicament non pourvoyeur d'érections prolongées.

Concernant les patients drépanocytaires, le traitement comporte une oxygénothérapie, des antalgiques, une hyperhydratation, une alcalinisation et éventuellement, des échanges transfusionnels de façon à diminuer l'hémoglobine $S$ à moins de $30 \%$ en complément du traitement médical $[9$, 18].

En cas de leucémies, la chimiothérapie en urgence reste le traitement de référence [1].

\section{Pronostic}

Concernant les résultats fonctionnels, le taux de succès est faible dans notre série, avec seulement $42,9 \%$ de bons résultats toutes techniques confondues, comparées aux autres séries de la littérature qui rapportent 50 à $60 \%$ de succès à distance (Tableau 7). En fait, le pronostic sexuel de ces malades dépend de plusieurs facteurs :

- Le délai de prise en charge parait comme le facteur le plus important. Plus ce délai est long, plus le risque dysfonction sexuelle définitive est élevé. En effet, $66,7 \%$ de nos patients traités dans les 72 premières heures ont récupéré une érection normale, alors que seulement $25 \%$ des malades traités au-delà de ce délai ont eu une évolution favorable. Ceci concorde avec la série de Winter et Mcdowell [21] où ces taux sont respectivement de 60 et $46 \%$.

- L'âge parait être également un facteur déterminant. La récupération d'une érection normale est d'autant moins facile que le sujet est plus avancé en âge. En effet, elle est de $76,9 \%$ chez les patients de moins de 40 ans et de $37,5 \%$ seulement au-delà de cet âge.

- Enfin, la technique opératoire utilisée semble elle aussi influencer les résultats fonctionnels lointains. Les meilleurs résultats obtenus d'emblée seraient observés avec la technique d'Al Ghorab (80\%) et à moindre degré avec l'anastomose caverno-spongieuse périnéale $(60 \%)$. Toutefois, ces conclusions sont basées sur des études comparatives sur une seule variable qui est la technique opératoire sans tenir compte des autres facteurs pronostiques.

En somme, le risque dysfonction érectile définitive demeure élevé, avoisinant les $50 \%$, dans les différentes séries de la littérature. Cette dysfonction érectile séquellaire est de très mauvais pronostic et sa correction est très difficile à réaliser car la fibrose caverneuse devient irréversible après 72 heures d'évolution. En effet, elle répond mal aux différents traitements médicaux, en particulier aux injections intracaverneuses de drogues vaso-actives, et seule la mise en place chirurgicale de prothèse pénienne permettra de remédier à cette complication. Par ailleurs, même la pose de prothèses dans de tels cas n'est pas pour autant aisée à cause de la fibrose importante des corps caverneux qui impose un forage pour pouvoir aménager l'espace nécessaire $[7,19]$.

\section{Prévention}

Le meilleur traitement du priapisme veineux reste la prévention. Les modalités de prise en charge d'une érection prolongée, de façon précoce, en particulier lors de l'utilisation des traitements facilitateurs et surtout inducteurs de l'érection, doivent être parfaitement appris aux patients [1]. 
Tableau 7 : résultats fonctionnels des différents traitements chirurgicaux dans la littérature.

\begin{tabular}{|c|c|c|c|c|}
\hline Auteur & $\begin{array}{c}\text { Nbre } \\
\text { de cas }\end{array}$ & Type d'intervention & $\begin{array}{c}\text { Age / } \\
\text { Délai ttt }\end{array}$ & $\begin{array}{l}\text { Récupération } \\
\text { sexuelle }\end{array}$ \\
\hline $\begin{array}{l}\text { Lund } \\
\text { et Ebbehoj [13] }\end{array}$ & 18 & $\begin{array}{l}\begin{array}{l}\text { Fistule spongio-caverneuse à ciel } \\
\text { fermé }\end{array}\end{array}$ & - & $61 \%$ \\
\hline \multirow{2}{*}{$\begin{array}{l}\text { Khoriaty } \\
\text { et Schick[12] }\end{array}$} & \multirow{2}{*}{138} & $\begin{array}{c}\text { Anastomose saphéno- } \\
\text { caverneuse }\end{array}$ & - & $61 \%$ \\
\hline & & Anastomose caverno-spongieuse & $\begin{array}{l}<40 \text { ans } \\
>40 \text { ans }\end{array}$ & $\begin{array}{l}73 \% \\
39 \% \\
\end{array}$ \\
\hline $\begin{array}{l}\text { Pohl } \\
\text { et al. [16] }\end{array}$ & 149 & Toutes techniques & & $54,6 \%$ \\
\hline \multirow{3}{*}{$\begin{array}{l}\text { Winter } \\
\text { et McDowell [21] }\end{array}$} & \multirow{3}{*}{105} & Fistule spongio-caverneuse & - & $55 \%$ \\
\hline & & Anastomose caverno-spongieuse & $\begin{array}{l}<72 \text { heures } \\
>72 \text { heures }\end{array}$ & $\begin{array}{l}50 \% \\
46 \%\end{array}$ \\
\hline & & $\begin{array}{l}\text { Anastomose saphéno- } \\
\text { caverneuse }\end{array}$ & - & $60 \%$ \\
\hline $\begin{array}{l}\text { Ravery } \\
\text { et al. [17] }\end{array}$ & 15 & Toutes techniques & - & $53,3 \%$ \\
\hline $\begin{array}{l}\text { Ben Chekroun } \\
\text { et al. [2] }\end{array}$ & 16 & Toutes techniques & - & $63 \%$ \\
\hline Falandry [9] & 56 & Toutes techniques & _ & $33,3 \%$ \\
\hline Falandry [10] & 26 & $\begin{array}{l}\text { Ponction-drainage balanique à } \\
\text { ciel ouvert }\end{array}$ & - & $65,3 \%$ \\
\hline Notre série (2007) & 21 & Toutes techniques & _ & $42,9 \%$ \\
\hline
\end{tabular}

ttt : du traitement.

D'autre part, la cessation rapide des crises de priapisme intermittent (à rechute) chez les patients drépanocytaires est primordiale permettant d'éviter la survenue de véritable crise de priapisme. Les mesures préventives associent une prise orale quotidienne d'étiléphrine, une oxygénothérapie nocturne à domicile, et si besoin, des IIC du même produit [18].

\section{CONCLUSION}

Le priapisme est une urgence uro-andrologique majeure dont le traitement s'impose sans délai avant que ne s'installent les lésions fibreuses définitives des corps caverneux responsables d'une dysfonction érectile irréversible. Le traitement médical peut être efficace dans les formes précoces mais il ne faut pas laisser passer l'heure de la chirurgie. Le pronostic sexuel dépend essentiellement du délai de prise en charge et de l'âge.

\section{REFERENCES}

1. AMSELLEM D., GUETTA T., ARVIS G. : Traitement médical et chirurgical du priapisme. Encycl. Méd. Chir. (Elsevier, Paris), Techniques chirurgicales. Urologie 41-457, 1997, $8 \mathrm{p}$.

2. BEN CHEKROUNA., LACHKARA., SOUMANAA., FARIH M.H. : Le priapisme de l'adulte. A propos de 16 cas. Ann. Urol., 1998, 32 : 103-106.
3. BONDIL P., DESCOTTES J.L., SALTIA., SABBAGH R., HAMZA T.: Traitement médical du priapisme veineux à propos de 46 cas : ponction, détumescence pharmacologique ou réfrigération pénienne? Prog. Urol., 1997, $7: 433-441$.

4. BRICHART N., DELAVIERRE D., PENEAU M., IBRAHIM H., MALLEK A. : Priapisme sous neuroleptiques. A propos de quatre patients. Prog. Urol., 2008, sous presse.

5. CHERIAN J., RAO A.R., THWAINI A., KAPASI F., SHERGILL I.S., SAMMAN R. : Medical and surgical management of priapism. Postgrad. Med. J., 2006, 82 : 89-94.

6. DEVAUX P., MIMOUN S. : Prostaglandine E1 dans le traitement de l'impuissance érectile. Efficacité et tolérance comparées selon différentes étiologies. J. Urol., 1994, 100 : 17-22.

7. DURAZI M.H., JALAL A.A. : Penile prosthesis implantation for treatment of postpriapism erectile dysfunction. J. Urol., 2008, 5 : 115-119.

8. ELAND I.A., VAN DER LEI J., STRICKER B.H.C., STURKENBOOM M.J.CM. : Incidence of priapism in the general population. Urology, 2001, $57:$ : 970-972.

9. FALANDRY L. : Priapisme : traitement et résultats. A propos d'une série personnelle de 56 cas : Prog. Urol., 1999, 9 : 496-501.

10. FALANDRY L. : Traitement chirurgical du priapisme par la ponctiondrainage balanique à " ciel ouvert » sur 26 patients de race noire. Ann. Urol., 2001, 35 : 172-177.

11. HSIAO H.L., WANG C.J., LI W.M., CHANG T.H., LI Y.C., HUANG C.H.: The Winter procedure and management for prolonged low-flow priapism : a case report. Kaohsiung J. Med. Sci., 2007, $23: 531-535$. 
12. KHORIATY N., SCHICK E. : La chirurgie du priapisme. J. Urol., $1980,86: 283-291$.

13. LUND K., EBBEHOJ J. : Results of glando-cavernous anastomosis in 18 cases of priapism. Scand. J. Plast. Reconstr. Surg., 1980, 14: 269-272.

14. MACALUSO J.N., SULLIVAN J.W. : Priapism : revue of 34 cases. Urology, 1985, $26: 233-236$.

15. MEIJER B., BAKKER H.R. : Management of priapism in the newborn. Urology, 2003, $61: 224 x v i-x v i i i$.

16. POHL J., POTT B., KLEINHANS G. : Priapism : a three-phase concept of management according to arteriolgy and prognosis. $\mathrm{Br}$. J. Urol., 1986, 58 : 113-118.

17. RAVERY V., DELAMS V., RESTREPO F., AMAR E., HERMIEN J.F., BOCCON-JIBOD L. : Priapisme de l'adulte : à propos de 15 cas. Prog. Urol., 1995, $5:$ 697-700.

18. ROUPRET M., BELEY S., TRAXER O. et al. : Prise en charge du priapisme chez les patients drépanocytaires. Prog. Urol., 2005, 15: 392-397.

19. UPADHYAY J., SHEKARRIZB., DHABUWALA C.B. : Penile implant for intractable priapism associated sickle cell disease. Urology, 1998, $51: 638-639$.

20. VILKE G.M., HARRIGAN R.A., UFBERG J.W., CHAN T.C. : Emergency evaluation and treatment of priapism. J. Emerg. Med., 2004, $26: 325-329$.

21. WINTER C.C., MCDOWELL G. : Experience with 105 patients with priapism : update review of all aspects. J. Urol., 1988, $140: 980-983$.

Manuscrit reçu : juillet 2008 ; accepté septembre 2008.

\begin{abstract}
Venous priapism : treatment and prognosis based on a series of 26 cases.

HADJ SLIMEN Mourad, FAKHFAKH Hammadi, GASSARA Mohamed, Sofiane KECHAOU, Ali BAHLOUL, Mohamed Nabil MHIRI
\end{abstract}

Introduction : Priapism is a prolonged, painful and persistent erection, unrelated to sexual stimulation. The management of priapism is complex and requires various medical or surgical treatments. The authors review the aetiology, treatment and outcome of priapism.

Material and methods : 26 cases of low-flow priapism were collected over a period of 23 years (1985-2007), corresponding to 22 adults and 4 children (average age: 32 years) with various types of priapism. The time to appropriate management after onset of priapism varied according to the aetiology and the time to consultation (range: 16 hours to 15 days).

Results : Complete and persistent detumescence for the first 24 hours was obtained in $\mathbf{8 4 \%}$ of cases, sometimes with multiple successive procedures. No case of early recurrence was observed. 21 patients were regularly followed for a period of 3 months to 10 years (mean follow-up: 2 years). There were $9(42.9 \%)$ successes (recovery of normal erectile function), 4 partial results $(19 \%)$ and $9(30.1 \%)$ failures (complete erectile dysfunction).

Conclusion : Low-flow priapism must be considered to be a serious andrological emergency. Medical treatment can be effective in early forms, but surgery must not be delayed to prevent permanent erectile dysfunction secondary to extensive corpora cavernosa fibrosis.

Key words: priapism, pharmacological erection, aetiologies, treatment, progression 